Proceedings of the XIII National School of Superconductivity, Lạdek Zdrój 2007

\title{
Spin Polarons in Weakly Doped Antiferromagnets: Experimental Evidence Obtained for Cuprates
}

\author{
P. Wróbel ${ }^{a}$, W. Suleja ${ }^{a}$ ANd R. $\operatorname{Eder}^{b}$
}

${ }^{a}$ Institute for the Low Temperature and Structure Research

P.O. Box 1410, 50-950 Wrocław 2, Poland

${ }^{b}$ Forschungszentrum Karlsruhe, IFP

P.O. Box 3640, D-76021 Karlsruhe, Germany

The mechanism of spin polaron formation in moderately doped cuprates is discussed. These objects represent holes embedded into heavy clouds formed by spin fluctuations. Wave functions of spin polarons are spatially confined due to the increase in the exchange energy which is induced by hole motion giving rise to the creation of spin fluctuations. These wave functions are eigenstates of an "unperturbed" Hamiltonian which is defined by processes responsible for the tendency toward confinement. The eigenstates transform according to different irreducible representations of the point group reflecting the symmetry of the problem. Thus, the spin polarons being local wave functions resemble orbital states. The spectrum of optical conductivity in the mid-infrared range is determined by transitions between $s$-wave and $p$-wave spin polarons. The hybridization between different spin polarons which is induced by some high order processes gives rise to the formation of energy bands. The pronounced transfer of the spectral weight between different bands is induced by the coupling between spin fluctuations created by the hopping hole and local quantum fluctuations in the empty antiferromagnetic background from which an electron has been rejected, for example during the photoemission process. The form of the energy dispersion for spin polarons gives rise to the formation of a small Fermi surface at the low hole-doping range. These three above mentioned phenomena were observed in cuprates which seems to confirm the spin polaron scenario. The discussion of related experiments is the additional objective of this paper.

PACS numbers: 71.27.+a, 71.10.Fd, 74.72.--h

\section{Introduction}

Having in mind the cuprate phase diagram, it is natural to suppose that the microscopic origin of high temperature superconductivity (HTSC) hinges on how quasiparticles are formed in a background of strong antiferromagnetic (AF) interactions. A natural starting point for the research on this subject is to learn how a single hole behaves in a half-filled AF system. This goal has been achieved by per- 
forming angle-resolved photoelectron spectroscopic (ARPES) measurements on the half-filled cuprates. Parent HTSC compounds such as $\mathrm{Sr}_{2} \mathrm{CuO}_{2} \mathrm{Cl}_{2} \mathrm{Ca}_{2} \mathrm{CuO}_{2} \mathrm{Cl}_{2}$ $[5,6], \mathrm{Nd}_{2} \mathrm{CuO}_{4}[7]$, and lightly doped $\mathrm{La}_{2} \mathrm{CuO}_{4}[8]$ have been studied.

In the recent and the most detailed study of insulating systems the compound $\mathrm{Ca}_{2} \mathrm{CuO}_{2} \mathrm{Cl}_{2}$ has been analyzed [6]. Due to the high resolution of experimental facilities a new dispersive feature have been found at higher binding energies along the $\Gamma$ to $(\pi, \pi)$ cut. The already known strongly renormalized structure with the dispersion of about $0.35 \mathrm{eV}$ has been observed at lower binding energies. The high energy part reveals a much more pronounced dispersion. The transfer of spectral weight from the low energy feature to higher energies occurs somewhere near the middle of the $(\pi, \pi)$ to $\Gamma$ cut. Quite recently, similar behavior has been also found in doped compounds [9-13].

As we will demonstrate later, a phenomenon directly related to the high-energy feature seen in the ARPES spectra is the so-called mid-infrared band observed during the measurements of the in-plane optical conductivity. Those measurements showed that in doped materials the energy region below the charge-transfer gap is filled with states at the expense of the spectral weight associated with the charge-transfer excitations in the undoped counterpart [14-16]. Recently, the optical spectroscopy combined with the derivative technique used for the high $T_{\mathrm{c}}$ cuprate $\mathrm{Bi}_{2} \mathrm{Sr}_{2} \mathrm{CaCu}_{2} \mathrm{O}_{8}$ gave additional evidence for the existence of the energy scale which may be attributed to the mid-infrared band [17].

Another relevant issue concerning unconventional behavior of cuprates is the origin of the differences in the physical properties of these systems doped to opposite sides of the superconducting region. On the right side of the superconducting dome, in the overdoped regime, the systems behave as a reasonably standard metal, with a large Fermi surface $[18,19]$. On the left side of the dome however, in the underdoped regime, cuprates are highly anomalous. For many years, instead of a closed Fermi surface only disconnected arcs have been observed [20]. Recently, the observation of quantum oscillations in the electrical resistance of the oxygen-ordered copper oxide $\mathrm{YBa}_{2} \mathrm{Cu}_{3} \mathrm{O}_{6.5}$ has been reported [21]. This observation indicates the existence of a well-defined Fermi surface in the ground state of underdoped copper oxides, once superconductivity is suppressed by a magnetic field. A Fermi surface made of small pockets has been revealed by the low oscillation frequency as the function of inverse field. This shape is in contrast to the large cylinder Fermi surface characteristic of the overdoped regime.

\section{Spin polaron approach}

The spin polaron (SP) approach is related to the so-called "string" scenario which has been developed since late sixties [22-27]. The string and SP scenarios are based on the following physical mechanism [28]. The hole motion in the AF gives rise to the creation of spin fluctuations which are defects in the underlying magnetic background. These defects lie on chains formed on paths along which 
the hole has moved. The length of such chains is in principle proportional to the increase in the exchange energy. Thus, the chains act on the hole as strings. The hole which can retrace the path to its starting point behaves as a particle in the potential well with the center on the initial position of the hole. A Hamiltonian for the problem of the hole in the potential well originating with the string effect has eigenstates with well defined energies. These states are related with a well defined modification of the AF background, thus they can be interpreted as $\mathrm{AF}$ SPs. There exists obvious similarity between SP states constructed in such a way and orbital states. They both possess an internal symmetry which may influence some spectroscopic measurements due to the existence of selection rules.

A conclusion which has been drawn from results obtained by means of approaches which originate with the string scenario [29, 28, 30-42] and also from results obtained at the same time by means of exact diagonalizations (EDs) [43-48] is that the Fermi surface of hole-doped cuprates, which are doped AF insulators, takes the form of four hole pockets located around points $( \pm \pi / 2, \pm \pi / 2)$. The formation of the hole pockets and of the small Fermi surface which is topologically different from the large conventional Fermi surface seen in overdoped systems has been very recently confirmed [21]. Some preliminary evidence for the scenario of the small Fermi surface in underdoped cuprates was also found in the results of earlier ARPES measurements [49]. The quasiparticle strength is weak at some parts of the hole pocket edge and the spectral weight in general is strongly momentum dependent, which explains the difficulty of identifying the small closed Fermi surface. Such a strong momentum dependence which was also observed in results of EDs [46] can be attributed to the interaction with spin fluctuations and can be explained by means of the SP approach $[50,51]$.

The propagation of the SP ground state determines the shape of the energy dispersion for the lowest energy "coherent band" which has been experimentally observed in an insulating cuprate by means of ARPES spectroscopy [52]. This dispersion was discussed by many researchers in the framework of the $t-J$ model $(t J \mathrm{M})[53,54]$. It seems that even some results of early analytical, semianalytical, and numerical analyses of that model [55-57, 47, 58] demonstrate the existence of excited SP states. The nature of these excited states and of the spectral-weight transfer between different bands formed by them is not only crucial for the proper interpretation of experimental results but is also important for the proper interpretation of results obtained by means of numerical calculations. Intriguing results of an ED performed for small clusters and published in early nineties seemed to indicate that already for two holes, which corresponds to $\approx 10 \%$ of doping in the case of 18-20 site clusters, a large electronic Fermi surface, compatible with the Luttinger theorem, is observed [59]. A careful analysis of those results suggested that they rather prove the validity of the rigid SP-band picture which implicates the existence of hole pockets even for the moderately hole doped $t J \mathrm{M}[60]$. The transitions in which different excited SP states with low symmetry are involved 
contribute substantially to the weight of the spectral function obtained by means of the ED. The "band structure" which emerges from numerical results can be explained in terms of the rigid band scenario provided that the transfer of the spectral weight is taken into account.

The idea of excited SP states was also applied some time ago [61] to explain the behavior of optical conductivity in the $t J \mathrm{M}$ in the context of measurements which demonstrated the formation of the mid-infrared band in the spectrum [14]. The existence of peaks in the spectral intensity at frequencies corresponding to a fraction of $1 \mathrm{eV}$ which corresponds to the position of the mid-infrared band was interpreted in terms of the transition to the $p$-like excited state of the SP. A simple calculation based on the string picture explaining the ED results semiquantitatively supported that suggestion. Later we will demonstrate, by means of a calculation based on the SP concept, the relation between the mid-infrared band observed in results of optical measurements and the high-energy anomaly observed in results of ARPES measurements. The results of this calculation will suggest that both features originate with the same branch of SP excitations.

We will use the $t J \mathrm{M}$ extended by terms enabling hopping to second and third nearest neighbors with hopping integrals $t^{\prime}$ and $t^{\prime \prime}$, respectively. We will apply standard values $t=0.35 \mathrm{eV}, t^{\prime}=-0.12 \mathrm{eV}, t^{\prime \prime}=0.08 \mathrm{eV}$, and $J=0.14 \mathrm{eV}$ chosen so as to reproduce the measured Fermi surface of hole doped cuprates for high doping levels.

\section{Energy bands and their spectral intensity}

The hybridization between SPs which are eigenstates of the unperturbed part of the full Hamiltonian for the $t J \mathrm{M}$ is induced by the remaining perturbed part. Respective terms representing this hybridization in the effective Hamiltonian $H_{\text {eff }}$ can be obtained by evaluating matrix elements of the perturbed part for different SP states. All details concerning the derivation of the effective Hamiltonian have been presented elsewhere [62]. By finding eigenstates and eigenvalues of $H_{\text {eff }}$ we obtain a band structure representing single-particle excitation energies.

In order to address the issue of spectral weight distribution observed by means of ARPES we analyze now the spectral function which is measured in experiments of that kind. The spectral intensity of a band $l$ is related with the strength of a pole at $E^{(l)}(\boldsymbol{k})$ in the appropriate Green function. That strength is given by the square of a matrix element,

$$
m^{(l)}(\boldsymbol{k})=\left\langle\Psi_{\downarrow}^{(l)}(-\boldsymbol{k})\left|c_{\boldsymbol{k}, \uparrow}\right| \Psi_{\mathrm{AF}}\right\rangle .
$$

In the formula (1) $\left|\Psi_{\downarrow}^{(l)}(\boldsymbol{k})\right\rangle$ is the $l$-th single-particle eigenstate of the operator $H_{\text {eff }}$ and $\left|\Psi_{\mathrm{AF}}\right\rangle$ stands for the AF state in which the photoemission process takes place, which means that we analyze electron emission from the undoped system. Wave vectors $\boldsymbol{k}$ and $-\boldsymbol{k}$ appear in (1) because the annihilation of an electron with a given momentum gives rise to the creation of a state with opposite momentum. The spin of the SP is also by definition opposite to the spin of the emitted electron. $\left|\Psi_{\mathrm{AF}}\right\rangle$ 
can be satisfactorily represented by a superposition of the Néel state and states in which local quantum spin fluctuations have been created. The main contribution

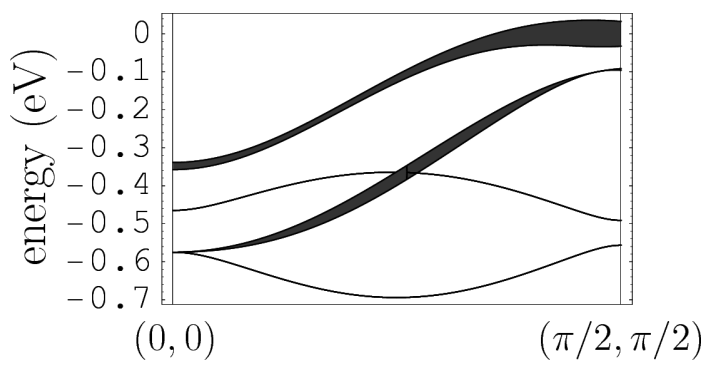

Fig. 1. Band structure obtained by solving the eigenvalue problem for the operator. The width of strips measured along vertical intervals connecting upper and lower edges of those strips represents the strength of poles at the energies $E^{(l)}(\boldsymbol{k})$ which are located in the middle of those intervals.

to (1) stems from the coupling between such local quantum spin fluctuations and strings states which are also spin fluctuations created by a hopping hole. The explicit calculation of coupling parameters will not be presented here. We merely mention that the local character of both string states and of quantum fluctuations in the AF gives rise to strong momentum dependence of spectral intensity for a given band. Partial results of the calculation have been presented in Fig. 1.

\section{Discussion and summary}

It turns out that the SP approach reproduces reasonably well the behavior of the measured intensity [62]. In both cases we observe the transfer of the spectral weight from a low energy feature to a feature formed at higher binding energies along the line connecting the point $(\pi / 2, \pi / 2)$ with the point $\Gamma$. The transfer occurs near the middle of the analyzed interval by about a half of $1 \mathrm{eV}$. In agreement with experimental data we also observe that the high energy feature disperses downward faster than the low energy feature towards the $\Gamma$ point where the intensity of both features weakens.

In our opinion the formation of the high energy feature provides strong arguments in favor of the SP scenario. The transfer of the spectral weight originates with the differences in contributions of local SP states with different symmetries to different bands and from strong momentum dependence of the pole strength, the form of which depends, in turn, on the contributions from different SP states. The outcome of our calculation suggests that the distribution of the spectral weight in weakly doped cuprates in the energy range of $1 \mathrm{eV}$ below the Fermi energy is determined by the coupling (1) between states representing local spin fluctuations in the AF state, and string states. 
States forming both bands with high weight along the line $\Gamma-(\pi / 2, \pi / 2)$ are even with respect to the reflection in the $(0,0)-(1,1)$ axis. That fact explains why $d$-wave SP states do not contribute to those bands. Due to symmetry properties of those SP states they can only contribute to states which are odd with respect to that reflection. The high-energy enhanced-weight band merges at the point $\Gamma$ with a low-weight band formed by states which are odd with respect to the reflection in the $(0,0)-(1,1)$ axis. The state at the junction point $\Gamma$ is doubly degenerate in that case and consists only of $p$-wave SPs. For that wave vector the low binding-binding energy state is built of $s$-wave SPs. That fact was used already some time ago in a theoretical analysis of optical spectra for doped AFs [61], because zero momentum states are involved in optical transitions. The finite frequency optical response is determined by the spectral distribution of the current operator, which obeys the dipole selection rule and couples $s$-wave states with $p$-wave states. In a simple calculation based on the SP approach the latter observation was used to calculate the optical conductivity. The outcome of that calculation agreed reasonably well with the results of an exact diagonalization, which allows to draw the conclusion that the formation of the mid-infrared band observed in the measured spectra of optical conductivity [14] should be attributed to the transitions between $s$-wave and $p$-wave SP states. The present analysis of the spectral function suggests that the states which contribute to the high-energy anomaly observed in ARPES spectra and the states which contribute to the optical mid-infrared band are related because in both cases they predominantly consist of $p$-wave SPs.

\section{Acknowledgments}

We thank R. Micnas for fruitful discussions. This work is dedicated to him on the occasion of his 60 th birthday.

\section{References}

[1] B.O. Wells, Z.-X. Shen, A. Matsuura, D.M. King, M.A. Kastner, M. Greven, R.J. Birgeneau, Phys. Rev. Lett. 74, 964 (1995).

[2] C. Kim, P.J. White, Z.-X. Shen, T. Tohyama, Y. Shibata, S. Maekawa, B.O. Wells, Y.J. Kim, R.J. Birgeneau, M.A. Kastner, Phys. Rev. Lett. 80, 4245 (1998).

[3] S. LaRosa, I. Vobornik, F. Zwick, H. Berger, M. Grioni, G. Margaritondo, Phys. Rev. B 56, R525 (1997).

[4] J.J.M. Pothuizen, R. Eder, N.T. Hien, M. Matoba, A.A. Menovsky, G.A. Sawatzky, Phys. Rev. Lett. 78, 717 (1997).

[5] F. Ronning, C. Kim, D.L. Feng, D.S. Marshall, A.G. Loeser, L.L. Miller, J.N. Eckstein, I. Bozovic, Z.-X. Shen, Science 282, 2067 (1998).

[6] F. Ronning, K.M. Shen, N.P. Armitage, A. Damascelli, D.H. Lu, Z.-X. Shen, L.L. Miller, C. Kim, Phys. Rev. B 71, 094518 (2005).

[7] N.P. Armitage, F. Ronning, D.H. Lu, C. Kim, A. Damascelli, K.M. Shen, D.L. Feng, H. Eisaki, Z.-X. Shen, Phys. Rev. Lett. 88, 257001 (2002).

[8] T. Yoshida, X.J. Zhou, T. Sasagawa, W.L. Yang, P.V. Bogdanov, A. Lanzara, Z. Hussain, T. Mizokawa, A. Fujimori, H. Eisaki, Z.-X. Shen, T. Kakeshita, S. Uchida, Phys. Rev. Lett. 91, 027001 (2003). 
[9] J. Graf, G.-H. Gweon, K. McElroy, S. Y. Zhou, C. Jozwiak, E. Rotenberg, A. Bill, T. Sasagawa, H. Eisaki, S. Uchida, H. Takagi, D.-H. Lee, A. Lanzara, Phys. Rev. Lett. 98, 067004 (2007).

[10] B.P. Xie, K. Yang, D.W. Shen, J.F. Zhao, H.W. Ou, J. Wei, S.Y. Gu, M. Arita, S. Qiao, H. Namatame, M. Taniguchi, N. Kaneko, H. Eisaki, K.D. Tsuei, C.M. Cheng, I. Vobornik, J. Fujii, G. Rossi, Z.Q. Yang, D.L. Feng, Phys. Rev. Lett. 98, 147001 (2007).

[11] T. Valla, T.E. Kidd, W.-G. Yin, G.D. Gu, P.D. Johnson, Z.-H. Pan, A.V. Fedorov, Phys. Rev. Lett. 98, 167003 (2007).

[12] Z.-H. Pan, P. Richard, A.V. Fedorov, T. Kondo, T. Takeuchi, S.L. Li, Pengcheng Dai, G.D. Gu, W. Ku, Z. Wang, H. Ding, arXiv:cond-mat/0610442 (2006).

[13] J. Chang, S. Pailhis, M. Shi, M. Mannsson, T. Claesson, O. Tjernberg, J. Voigt, V. Perez, L. Patthey, N. Momono, M. Oda, M. Ido, A. Schnyder, C. Mudry, J. Mesot, Phys. Rev. B 75, 224508 (2007).

[14] S. Uchida, T. Ido, H. Takagi, T. Arima, Y. Tokura, S. Tajima, Phys. Rev. B 43, 7942 (1991).

[15] S.L. Cooper, D. Reznik, A. Kotz, M.A. Karlow, R. Liu, M.V. Klein, W.C. Lee, J. Giapintzakis, D.M. Ginsberg, B.W. Veal, A.P. Paulikas, Phys. Rev. B 47, 8233 (1993).

[16] M. Grüninger, D. van der Marel, A. Damascelli, A. Erb, T. Nunner, T. Kopp, Phys. Rev. B 62, 12422 (2000).

[17] J. Hwang, E.J. Nicol, T. Timusk, A. Knigavko, J.P. Carbotte, Phys. Rev. Lett. 98, 207002 (2007).

[18] N.E. Hussey, M. Abdel-Jawad, A. Carrington, A.P. Mackenzie, L. Balicas, Nature 425, 814 (2003).

[19] M. Platé, J.D.F. Mottershead, I.S. Elfimov, D.C. Peets, Ruixing Liang, D.A. Bonn, W.N. Hardy, S. Chiuzbaian, M. Falub, M. Shi, L. Patthey, A. Damascelli, Phys. Rev. Lett. 95, 077001 (2005); and references therein.

[20] M. Norman, H. Ding, M. Randeria, J.C. Campuzano T. Yokoya, T. Takeuchi, T. Takahashi, T. Mochiku, K. Kadowaki, P. Guptasarma, D.G. Hinks, Nature 392, 157 (1998).

[21] N. Doiron-Leyraud, C. Proust, D. LeBoeuf, J. Levallois, J.-B. Bonnemaison, R. Liang, D.A. Bonn, W.N. Hardy, L. Taillefer, Nature 447, 565 (2007).

[22] L.N. Bulaevskii, Sov. Phys. JETP 27F, 836 (1968).

[23] W.F. Brinkman, T.M. Rice, Phys. Rev. B 2, 1324 (1970).

[24] J.E. Hirsch, Phys. Rev. Lett. 59, 228 (1987).

[25] S.A. Trugman, Phys. Rev. B 37, 1597 (1988).

[26] B.I. Shraiman, E.D. Siggia, Phys. Rev. Lett. 60, 740 (1988).

[27] S. Schmitt-Rink, Phys. Rev. Lett. 60, 2793 (1988).

[28] R. Eder, K.W. Becker, Z. Phys. B 78, 219 (1990).

[29] C.L. Kane, P.A. Lee, N. Read, Phys. Rev. B 39, 6880 (1989).

[30] A. Ramšak, P. Prelovšek, Phys. Rev. B 42, 10415 (1990).

[31] P. Prelovšek, I. Sega, J. Bonča, Phys. Rev. B 42, 10706 (1990). 
[32] P. Fulde, Electron Correlations in Molecules and Solids, Springer Ser. Solid-State Sci., Vol. 100R, Springer, Berlin 1991.

[33] F. Marsiglio, A.E. Ruckenstein, S. Schmitt-Rink, C.M. Varma, Phys. Rev. B 43, 10882 (1991).

[34] G. Martinez, P. Horsch, Phys. Rev. B 44, 317 (1991).

[35] Z. Liu, E. Manousakis, Phys. Rev. B 45, 2425 (1992).

[36] A.L. Chernyshev, A.V. Dotsenko, O.P. Sushkov, Phys. Rev. B 49, 6197 (1994).

[37] V.I. Belinicher, A.L. Chernyshev, A.V. Dotsenko, O.P. Sushkov, Phys. Rev. B 51, 6076 (1995).

[38] J. Bała, A.M. Oleś, J. Zaanen, Phys. Rev. B 52, 4597 (1995).

[39] M. Vojta, K.W. Becker, Phys. Rev. B 54, 15483 (1996).

[40] N.M. Plakida, V.S. Oudovenko, P. Horsch, A.I. Liechtenstein, Phys. Rev. B 55, R11997 (1997).

[41] V.I. Belinicher, A.L. Chernyshev, V.A. Shubin, Phys. Rev. B 56, 3381 (1997).

[42] M. Vojta, K.W. Becker, Phys. Rev. B 57, 3099 (1998).

[43] J. Bonča, P. Prelovšek, I. Sega, Phys. Rev. B 39, 7074 (1989).

[44] J.A. Riera, Phys. Rev. B 40, 833 (1989).

[45] Y. Hasegawa, D. Poilblanc, Phys. Rev. B 40, 9035 (1989).

[46] K.J. von Szczepanski, P. Horsch, W. Stephan, M. Ziegler, Phys. Rev. B 41, 2017 (1990).

[47] E. Dagotto, R. Joynt, A. Moreo, S. Bacci, E. Gagliano, Phys. Rev. B 41, 9049 (1990).

[48] P.W. Leung, R.J. Gooding, Phys. Rev. B 52, R15711 (1995).

[49] F. Ronning, T. Sasagawa, Y. Kohsaka, K.M. Shen, A. Damascelli, C. Kim, T. Yoshida, N.P. Armitage, D.H. Lu, D.L. Feng, L.L. Miller, H. Takagi, Z.-X. Shen, Phys. Rev. B 67, 165101 (2003).

[50] R. Eder, K.W. Becker, Phys. Rev. B 44, 6982 (1991).

[51] O.P. Sushkov, G.A. Sawatzky, R. Eder, H. Eskes, Phys. Rev. B 56, 11769 (1997).

[52] B.O. Wells, Z.-X. Shen, A. Matsuura, D.M. King, M.A. Kastner, M. Greven, R.J. Birgeneau, Phys. Rev. Lett. 74, 964 (1995).

[53] K.A. Chao, J. Spałek, A.M. Oleś, Phys. Rev. B 18, 3453 (1978).

[54] F.C. Zhang, T.M. Rice, Phys. Rev. B 37, 3759 (1988).

[55] B.I. Shraiman, E.D. Siggia, Phys. Rev. Lett. 61, 467 (1988).

[56] B.I. Shraiman, E.D. Siggia, Phys. Rev. Lett. 62, 1564 (1989).

[57] T. Barnes, E. Dagotto, A. Moreo, E.S. Swanson, Phys. Rev. B 40, 10977 (1989).

[58] V. Elser, D.A. Huse, B.I. Shraiman, E.D. Siggia, Phys. Rev. B 41, 6715 (1990).

[59] W. Stephan, P. Horsch, Phys. Rev. Lett. 66, 2258 (1991).

[60] R. Eder, P. Wróbel, Phys. Rev. B 47, 6010 (1993).

[61] R. Eder, P. Wróbel, Y. Ohta, Phys. Rev. B 54, R11034 (1996).

[62] P. Wróbel, W. Suleja, R. Eder, Phys. Rev. B, accepted for publication. 